physician or hospital visit. To ensure incident gout, we required that all cases have $\geq 10$ years of prior registration without a gout diagnosis. Gout cases were matched 1:1 with controls on age, sex, and cohort entry time. Prior VTE events were excluded after matching. VTE was defined using ICD-9/ICD-10 codes plus use of oral anti-coagulant. We calculated incidence rate ratios (IRRs) and age, sex, and entry time matched multivariable hazard ratios (HRs) for the risk of VTE. Sensitivity analyses were conducted to assess for unmeasured confounders (e.g., obesity).

Results: Among 105,307 individuals with newly diagnosed gout (61\% male, mean age of $58 \mathrm{yrs}$ ), we observed 1,212 VTE events (mean follow-up time of $5.3 \mathrm{yrs}$ ). The fully adjusted HR was 1.27 (95\% Cl, 1.16-1.39) (Table 1). Our results remained significant in the sensitivity analyses (OR of 2.0 between gout and unmeasured confounder and a $50 \%$ prevalence for obesity). There were 437 incident VTE events within 3 yrs prior to the gout diagnosis. Compared to controls, during the 3 rd, 2nd, and 1st yrs before gout diagnosis, the fully adjusted HRs for VTE in patients with imminent gout were 1.51, 1.58 and 1.73. The corresponding HRs in the 1st, 2nd, 3rd, 4th, and 5th yrs after gout diagnosis were 1.54, 1.39, $1.38,1.37$, and 1.32 , respectively.

\begin{tabular}{lccccccc} 
Table 1 & $\begin{array}{c}\text { Gout } \\
(\mathrm{N})\end{array}$ & $\begin{array}{c}\text { Control } \\
(\mathrm{N})\end{array}$ & $\begin{array}{c}\text { No. of VTE } \\
\text { events }\end{array}$ & $\begin{array}{c}\text { IRRs } \\
(95 \% \mathrm{Cl})\end{array}$ & $\begin{array}{c}\text { Age-, sex-adjusted } \\
(95 \% \mathrm{Cl})\end{array}$ & $\begin{array}{c}\text { Fully adjusted } \\
(95 \% \mathrm{Cl})\end{array}$ \\
\cline { 3 - 6 } & & \multicolumn{6}{c}{ Gout Control } \\
\end{tabular}

*Adjusted for Charlson comorbidity index, cancer, alcoholism, hypertension, sepsis, trauma, healthcare utilization, NSAIDs, HRT, glucocorticoid and fractures, surgery within 1 year before entry cohort.

Conclusions: Patients with gout have an increased risk of VTE. The risk increases gradually before gout diagnosis, peaks in the year prior to gout diagnosis, and then progressively declines. Our findings suggest that hyperuricemia or gout associated inflammation may be a contributing factor for VTE.

Disclosure of Interest: None declared

DOI: 10.1136/annrheumdis-2017-eular.4146

\section{THU0441 SYNOVIAL FLUID LEUKOCYTE COUNT AND ITS ASSOCIATION WITH CRYSTAL DEPOSITION IN ASYMPTOMATIC HYPERURICEMIA: A PRELIMINARY REPORT}

M. Andrés ${ }^{1,2}$, J.A. Bernal ${ }^{2}$, M.D. Arenas ${ }^{3}$, E. Pascual ${ }^{2,4} .{ }^{1}$ Departamento de Medicina Clínica, Universidad Miguel Hernández; ${ }^{2}$ Seccion de Reumatologia, Hospital General Universitario de Alicante; ${ }^{3}$ Unidad de Nefrología, Hospital Vithas Perpetuo Socorro; ${ }^{4}$ Emeritus Professor, Universidad Miguel Hernández, Alicante, Spain

Background: Joint deposits of monosodium urate (MSU) crystals in asymptomatic hyperuricemia $(\mathrm{AH})$ may associate with more severe forms of atherosclerosis [1]. It likely relates with crystal-driven subclinical inflammation, a phenomenon well described in gout [2] but to date not assessed in $\mathrm{AH}$.

Objectives: To assess the synovial fluid (SF) leukocytes count in patients with $\mathrm{AH}$ depending on the presence of crystals.

Methods: Consecutive patients with AH were selected from nephrology clinics. Hyperuricemia threshold was established at serum uric acid (SUA) $\geq 7 \mathrm{mg} / \mathrm{dL}$; those on current urate-lowering therapy were excluded. Ultrasound (US) of knees, ankles and first metatarsophalangeal joints was performed blinded to clinical and laboratory data, in order to perform US-guided aspiration and obtain SF samples. These samples were analyzed in fresh by two observers, using a compensated polarized light microscope equipped with two viewing stations. The second observer was in addition unaware of US findings. The presence of leukocytes and crystals was established by consensus. SF leukocyte count was performed using a Neubauer counting camera. Mann-Whitney's $U$ and Kruskal-Wallis' $H$ were used to compare difference in leukocyte counts between groups.

Results: To end of January 2017, 30 patients have been assessed, with SF samples available from 27 (three showed no joint effusion at US). Median age was 70 years (p25-75 59.8-75.5), and 16 of them (60\%) were males. Median (p25-75) SUA and estimated glomerular filtration rate were $8.1 \mathrm{mg} / \mathrm{dL}$ (7.6-8.5) and $37.0 \mathrm{~mL} / \mathrm{min}(31.0-47.5)$, respectively. Seven (25\%) were on diuretics. After microscopy evaluation, MSU crystals were found in four patients (14.8\%), calcium pyrophosphate (CPP) crystals in five (18.5\%), and no crystals in $18(66.7 \%)$. Figure shows median leukocytes count according to crystal group: $200 / \mathrm{mm}^{3}$ (138-540) in MSU group; 60/ $\mathrm{mm}^{3}$ (50-235) in CPP group; and 30/ $\mathrm{mm}^{3}(10-53)$ in those with no crystal at SF. A statistically significant difference was found between the three groups $(p=0.001)$, and comparing MSU crystals and no crystals groups $(p<0.001)$; no difference was found between MSU and CPP groups $(p=0.111)$

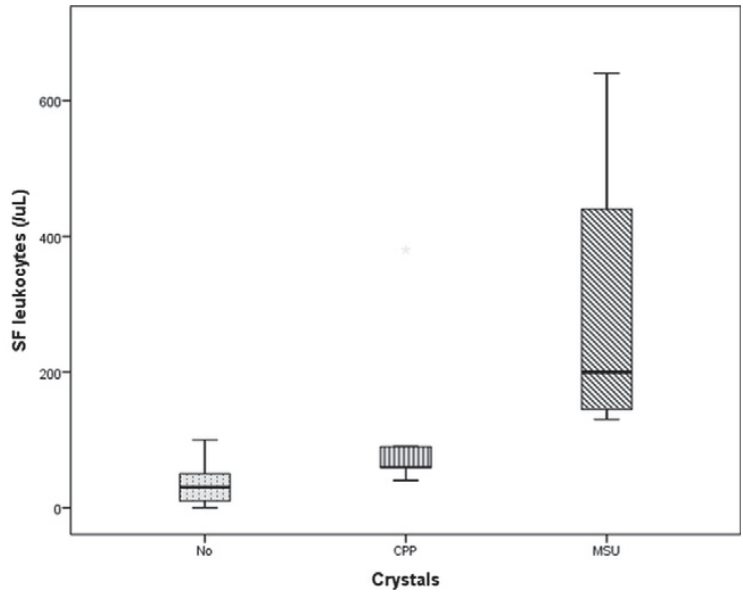

Conclusions: In a preliminary report, MSU crystal deposition in patients with $\mathrm{AH}$ leads to higher SF leukocyte count, indicating low-grade inflammation. Despite low numbers and being an ongoing study, this significant finding might contribute to explain the severe atherosclerosis data recently reported in $\mathrm{AH}$ patients with silent crystal deposits.

References:

[1] Arthritis Rheumatol 2016;68:1531.

[2] Arthritis Rheum 1991;34:141.

Disclosure of Interest: None declared

DOI: 10.1136/annrheumdis-2017-eular.3258

\section{THU0442 EHEALTH SUPPORTED COLLABORATIVE CARE MODEL FOR GOUT INVOLVING RHEUMATOLOGY, PHARMACY, AND DIETETICS: PROOF-OF-CONCEPT OBSERVATIONAL STUDY}

M.A. De Vera ${ }^{1,2}$, A. Howren ${ }^{1}$, N. Tsao ${ }^{1}$, H. Choi $^{3}$, A. Kydd ${ }^{4}$, R. Friesen ${ }^{5}$, K. Shojania ${ }^{2,4}$. ${ }^{1}$ Faculty of Pharmaceutical Sciences, University of British Columbia, Vancouver; ${ }^{2}$ Arthritis Research Canada, Richmond, Canada; ${ }^{3}$ Department of Medicine, Massachusetts General Hospital, Boston, United States; ${ }^{4}$ Division of Rheumatology, University of British Columbia; ${ }^{5}$ Providence Health, Vancouver, Canada

Background: Sub-optimal quality of care for gout, the most common inflammatory arthritis, has called for novel models to improve care and outcomes of patients. Objectives: Our objective was to evaluate the feasibility of the "Virtual Gout Clinic Study" (VGCS) a collaborative approach to gout management involving rheumatology, pharmacy, and dietetics that take advantage of eHealth, namely, shared access of electronic medical records (EMRs), to support this unique, decentralized model of care.

Methods: Design: We conducted a 1-year, proof-of-concept, longitudinal observational study with a target of 30 patients. Eligibility: Patients with gout were eligible if they were: 1) seen in 1 of 4 rheumatology practices with 8 rheumatologists participating across British Columbia, Canada; 2) experienced $\geq 1$ flare in the past year; 3 ) have a serum uric acid (SUA) level $>360 \mu \mathrm{mol} / \mathrm{L}$ within the previous 2 months. Follow-up: Within the VGCS, patients receive follow-up with their rheumatologist on an as needed-basis, monthly telephone consults from the study pharmacist, and 1 telephone consult with the dietician. In this decentralized model of care, health care professionals are not co-located but have access to the patient's EMR to facilitate remote communication and collaboration. Outcomes: Prospective data was collected at baseline, 3, 6, and 12 months. The primary outcome measure was SUA at 12-months, specifically below recommended target level of $360 \mu \mathrm{mol} / \mathrm{L}$. Secondary outcomes included patient reported outcome measures including the Compliance Questionnaire Rheumatology, Health Assessment Questionnaire Disability Index, and EQ-5D, as well as information on interventions provided within the VGCS.

Results: Overall, 33 individuals with gout participated in the VGCS. There were 22 males (88\%) and mean age was $60.8 \pm 15.3$ years. 29, 28, and 25 patients have completed follow-up at 3,6 , and 12 months respectively. At baseline, 22 (67\%) patients were prescribed urate-lowering therapy (ULT; 21 with allopurinol and 1 with febuxostat). The remaining 11 patients initiated ULT during the study period. Figure 1 shows mean SUA decreased over baseline, 3, 6, and 12 months from $451 \mu \mathrm{mol} / \mathrm{L}, 367 \mu \mathrm{mol} / \mathrm{L}, 356 \mu \mathrm{mol} / \mathrm{L}$, and $364 \mu \mathrm{mol} / \mathrm{L}$, respectively, with $67 \%$ of patients having SUA $<360 \mu \mathrm{mol} / \mathrm{L}$ at 12 months. A total of 126 pharmacist and 24 dietician consults have been provided. Pharmacist interventions included: uptritration of allopurinol dosage for 15 patients, restarting of ULT for 4 nonadherent patients, discontinuation of unnecessary medications for 12 patients, and renewals of prescription medications using solely electronic communications for 4 patients.

Conclusions: These findings provide evidence for the feasibility and effectiveness of the VGCS. With respect to feasibility, we successfully established the shared EMR framework between rheumatologists and study pharmacist and dietitian and demonstrated utility with respect to facilitating communication and collaborative 


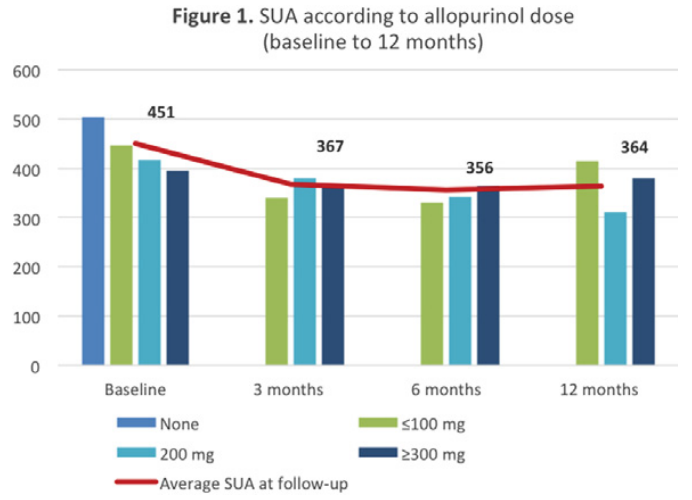

care between health care providers and providing outcomes data. With respect to effectiveness, findings show declining SUA over follow-up with over two-thirds of patients achieving target at the end of follow-up.

Disclosure of Interest: None declared

DOI: 10.1136/annrheumdis-2017-eular.4177

\section{THU0443 HYPERURICEMIA IS ASSOCIATED WITH INCREASED CORONARY ARTERY CALCIFICATION IN MEN BUT NOT WOMEN}

M.I. Dehlin ${ }^{1}$, P. Drivelegka ${ }^{1}$, V. Sigurdardottir ${ }^{1}$, O. Angerås ${ }^{2}$, L.T. Jacobsson ${ }^{1}$, H. Forsblad d'Elia ${ }^{1,3} \cdot{ }^{1}$ Dept of rheumatology and inflammation research, Institution of medicine, Sahlgrenska Academy, University of Gothenburg; ${ }^{2}$ Department of Cardiology, Sahlgrenska University Hospital, Gothenburg;

${ }^{3}$ Departments of Public Health and Clinical Medicine, Rheumatology, Umeå University, Umeå, Sweden

Background: Hyperuricemia is closely associated to cardiovascular disease although it has not been defintively established whether it is a marker or a causative agent. Serum uric acid (sUa) is strongly linked to the metabolic syndrome, hypertension ( $\mathrm{HT}$ ), dyslipidemia (DL) and higher BMI and higher levels are seen in men compared to women. Coronary artery calcification (CAC) is associated with future risk of atherosclerotic CV events in addition to these traditional cardiovascular risk factors (CVRF). CACs are present in atherosclerotic arteries and can be quantified and scored non-invasively by computed tomography. The Swedish CArdioPulmonarybiolmage Study (SCAPIS) extensively characterizes a Swedish cohort of 30000 men and women aged between 50 and 64 years. A comprehensive pilot study in 1111 individuals was completed in 2012. In this pilot study we have examined the relation between CACs and SUa

Objectives: Examine the association bewteen sUa and CAC in men and women seperately.

Methods: In the SCAPIS pilot study we identified 1106 (552 males) individuals who were screened for traditional CVDRFs, such as HT, DL, diabetes mellitus (DM), smoking, physical activity (PA), educational level (EDU), BMI, high sensitive CRP (hsCRP). CACs, reflecting calcification of coronary arteries, was determined according to Agatston ${ }^{1}$. We measured sUa and related quartiles to CACs with multiple logistic regression analyses adjusting for traditional CVDRFs. CAC was defined positive if $\geq 1$.

Table 1. Baseline characteristics of the study population divided by sex

\begin{tabular}{lcc}
\hline Variables & Male, $\mathrm{n}=552$ & Female, $\mathrm{n}=556$ \\
\hline Age, years, mean, (SD) & $57.8(4.5)$ & $57.6(4.3)$ \\
BMI, mean, (SD) & $27.7(4.0)$ & $26.9(3.7)$ \\
hsCRP, (mean, SD) & $2.3(3.3)$ & $2.4(3.7)$ \\
CAC positive, (\%) & $305(55 \%)$ & $142(26 \%)$ \\
Smoking status, never & $226(41 \%)$ & $249(45 \%)$ \\
Smoking status, previous & $242(44 \%)$ & $222(40 \%)$ \\
Smoking status, active & $82(15 \%)$ & $81(15 \%)$ \\
Hypertension (\%) & $178(32 \%)$ & $190(34 \%)$ \\
Diabetetes mellitus (\%) & $45(8 \%)$ & $23(4 \%)$ \\
Dyslipidemia (\%) & $160(29 \%)$ & $128(23 \%)$ \\
\hline
\end{tabular}

Table 2. Quartiles of sUa and age as predictors for presence of CAC (>0 CACs score) in male and female in multivariate logistic regression analyses adjusted for age, smoking, BMI, DM, DL, $\mathrm{HT}$, hsCRP, EDU and PA

\begin{tabular}{|c|c|c|c|c|c|}
\hline \multirow[t]{2}{*}{$\mathrm{sUa}, \mu \mathrm{mol} / \mathrm{L}$, } & \multicolumn{2}{|l|}{ Male } & \multirow[t]{2}{*}{$\mathrm{sUa}, \mu \mathrm{mol} / \mathrm{L}$, } & \multicolumn{2}{|l|}{ Female } \\
\hline & $\begin{array}{c}\text { Odds ratio, } \\
\text { multivariate }^{\star},(\mathrm{Cl})\end{array}$ & $p$-value & & $\begin{array}{c}\text { Odds ratio, } \\
\text { multivariate }^{\star},(\mathrm{Cl})\end{array}$ & $p$-value \\
\hline 31-305, Ref & & & 143-229, Ref & & \\
\hline $306-350$ & $2.1(1.2-3.7)$ & 0.01 & 230-262 & $0.9(0.5-1.7)$ & 0.7 \\
\hline $351-404$ & $1.8(1-3.3)$ & 0.04 & $263-305$ & $1(0.5-2)$ & 0.9 \\
\hline $405-665$ & $2(1-3.8)$ & 0.04 & $306-702$ & $1.2(0.6-2.3)$ & 0.6 \\
\hline \multicolumn{6}{|l|}{ Age 50-55, Ref } \\
\hline Age $56-60$ & $2.2(1.4-3.6)$ & 0.001 & & $3.6(2-6.5)$ & $<0.0001$ \\
\hline Age $61-66$ & $3.8(2.2-6.3)$ & $<0.0001$ & & $4.3(2.4-7.9)$ & $<0.0001$ \\
\hline
\end{tabular}

Results: Age, BMI, smoking status, hsCRP, HT and DL showed no differences between sex while presence of CAC and diabetes was twice as common in men (Table 1). The three upper quartiles of sUa, $(>306 \mu \mathrm{mol} / \mathrm{L})$, all significantly $(\mathrm{p}<0.05)$ predicted presence of CACs in men even adjusting for HT, DL, DM, smoking, PA, EDU, BMI, hsCRP and age in multivariate logistic regression, but not in women (Table 2).

Conclusions: Higher levels of $\mathrm{sUa}$ is associated with presence of CACs in men but not in women. This may merely reflect the earlier onset of atherosclerosis in men or possibly suggest biological differences in the effect of sUa on calcification of coronary arteries between sexes.

References:

[1] Agatston AS et al Quantification of coronary artery calcium using ultrafast computed tomography J Am Coll Cardiol 1990.

Disclosure of Interest: None declared

DOI: 10.1136/annrheumdis-2017-eular.2714

\section{THU0444 INTEGRATED SAFETY OF LESINURAD, A NOVEL URIC ACID REABSORPTION INHIBITOR FOR THE TREATMENT OF GOUT}

M.A. Becker ${ }^{1}$, R.T. Keenan ${ }^{2}$, P.P. Khanna ${ }^{3}$, R. Malamet ${ }^{4}$, K. Bos ${ }^{4}, \mathrm{~J} \mathrm{Li}^{4}$ J. Hu ${ }^{5}$, W.B. White ${ }^{6} .{ }^{1}$ University of Chicago, Chicago; ${ }^{2}$ Duke University School of Medicine, Durham; ${ }^{3}$ University of Michigan, Ann Arbor; ${ }^{4}$ AstraZeneca Pharmaceuticals, Wilmington; ${ }^{5}$ Formerly Ardea Biosciences, Inc, San Diego ${ }^{6}$ University of Connecticut School of Medicine, Farmington, United States

Background: Lesinurad is a selective uric acid reabsorption inhibitor recently approved at $200 \mathrm{mg}$ daily in combination with a xanthine oxidase inhibitor (XOI) for treatment of hyperuricemia associated with gout in patients who have not achieved target serum uric acid on an XOI (allopurinol or febuxostat) alone.

Objectives: To investigate the safety profile of lesinurad (LESU), we integrated safety data based on: (1) 3 large, pivotal, placebo-controlled, 12-month phase III (core) trials evaluating LESU $200 \mathrm{mg}$ and LESU $400 \mathrm{mg}$ in combination with an $\mathrm{XOI}$; and (2) 2 extension studies, in which LESU-treated patients continued to receive $\mathrm{LESU}+\mathrm{XOI}$ at the same dose and initially placebo-treated patients were randomized to receive LESU $200 \mathrm{mg}$ or LESU $400 \mathrm{mg}$ in addition to the XOI provided in the preceding core trial.

Methods: Safety data were pooled from the 3 core studies and 2 12-month extension studies using descriptive statistics for patients receiving $>1$ dose of study medication. To adjust for varying treatment durations, treatment-emergent adverse events (TEAEs) are expressed as exposure-adjusted incidence rates (number of subjects with events per 100 person-years [PY]).

Results: In the core studies, adverse event rates were comparable for $\mathrm{XOI}$ alone and LESU $200 \mathrm{mg}+$ XOI groups for any TEAEs, serious TEAEs, and TEAEs leading to discontinuation (Table 1). Adverse event rates were higher with LESU $400 \mathrm{mg}+$ XOI. Major adverse cardiovascular event (MACE) rates, which included cardiovascular death, myocardial infarction, or stroke, in the core studies were 0.71 $(95 \% \mathrm{Cl} 0.15,2.08), 0.96(0.26,2.47)$, and $1.94(0.84,3.82)$ per $100 \mathrm{PY}$ for $\mathrm{XOI}$

Table 1. Pooled Analysis of Exposure-Adjusted Adverse Event Incidence Rates in 3 Piwotal Placebo-Controlled 12-Month Phase III Studies Evaluating Lesinurad $200 \mathrm{mg}$ and $400 \mathrm{mg}$ in Combination with Xanthine Oxidase Inhibitors

\begin{tabular}{|c|c|c|c|}
\hline \multirow[b]{2}{*}{ Adverse Event Category [n (rate)] } & \multirow{2}{*}{$\begin{array}{l}\text { XOl alone } \\
(N=516) \\
(P Y=408.5)\end{array}$} & \multicolumn{2}{|c|}{ LESU $200 \mathrm{mg}+$ LESU $400 \mathrm{mg}$ t } \\
\hline & & $\begin{array}{c}X O 1 \\
(N=511) \\
(P Y=396.3)\end{array}$ & $\begin{array}{c}x O I \\
(N=510) \\
(P Y=390.5)\end{array}$ \\
\hline AMY TEAE & $363(88.9)$ & $386(97.4)$ & $407(104.2)$ \\
\hline Amy TEAE with RCTC toxicity Grade 3 or 4 & $48(11.8)$ & $52(13.1)$ & $67(127.2)$ \\
\hline Any TEAE possibly related to randomized study medication & $80(19.6)$ & $98(24.7)$ & $118(30.2)$ \\
\hline Any TEAE possibly related to XOI & $52(12.7)$ & $49(12.4)$ & $66(16.9)$ \\
\hline Any TEAE possibly related to prophylaxis & S2(12.7) & $56(14.1)$ & $61(25.6)$ \\
\hline Any serious TEAE & $29(7.1)$ & $24(6.1)$ & $44(11.3)$ \\
\hline Any fatal TEAE & o(0) & $2(0.5)$ & $3(0.8)$ \\
\hline AMY TEAE leading to randomized study medication discontinuation & $28(6.9)$ & $32(8.1)$ & $48(12.3)$ \\
\hline
\end{tabular}

Table 2. Pooled Analysis of Exposure-Adjusted Adverse Event Incidence Rates in 3 Piwotal Placebo-Controlled 12-Month Phase III Studies +2 Extension Studies Evaluating Lesinurad $200 \mathrm{mg}$ and $400 \mathrm{mg}$ in Comblnation with Xanthine Oxidase Inhibitors

\begin{tabular}{|c|c|c|}
\hline Adverse Event Category [n (rate)] & $\begin{array}{c}\text { LESU } 200 \mathrm{mg} \\
+\times 01 \\
(\mathrm{~N}=666) \\
(\mathrm{PY}=926.5)\end{array}$ & $\begin{array}{c}\text { LESU } 400 \mathrm{mg} \\
\text { XO1 } \\
(\mathrm{N}=666) \\
(P Y=917.9)\end{array}$ \\
\hline Any TEAE & $531(57.3)$ & $552(60.1)$ \\
\hline Any TEAE with RCTC toxicity Grade 3 or 4 & $93(10.0)$ & 115 (12.5) \\
\hline Any TEAE possibly related to randomized study medication & $158(17.1)$ & $187(20.4)$ \\
\hline Any TEAE possibly related to $X O l$ & $n[8.3\}$ & $101(11.0)$ \\
\hline Any TEAE possibly related to prophylaxis & $76\{8.21$ & $81(8.8)$ \\
\hline Any serious TEAE & $63(6.8)$ & $84(9.2)$ \\
\hline Any fatal TEAE & $7(0.8)$ & $7(0.8)$ \\
\hline Any TEAE leading to randomized study medication discontinuation & $68(7.3)$ & $86(9.4)$ \\
\hline
\end{tabular}

